\title{
Retraction: How COVID-19 Brought Indian Wedding Industry to a Halt?
}

\author{
Ritik Ranjan Gupta, Ravi Kumar Arya, Jatin Kumar, Tanay Shubham
}

National Institute of Technology Delhi, India

This paper was retracted by JINAV Journal on 03 February 2021. This paper was published in another journal (International Journal of Management and Humanities (IJMH), Volume 5 Issue 5, January 2021). 